LETTER TO JMG

\title{
No association between a previously reported OLR 1 3' UTR polymorphism and Alzheimer's disease in a large family sample
}

\author{
L Bertram, M Parkinson, K Mullin, R Menon, D Blacker, R E Tanzi
}

J Med Genet 2004;41:286-288. doi: 10.1136/jmg.2003.016980

$\mathrm{R}$ ecently, two studies ${ }^{12}$ reported independent evidence of genetic association between a 3' UTR single nucleotide polymorphism ( SNP; $r$ s 1050283, also known as "+1073") in the oxidised LDL receptor 1 gene (OLRI) on chromosome 12pl3.2 and risk for Alzheimer's disease (AD). The implied chromosomal area is a highly promising AD candidate region because both genetic linkage and association studies have reported significant signals to two different locations separated by about $40 \mathrm{Mb}$ (about $44 \mathrm{cM}$ ): the more proximal region is located near $10 \mathrm{Mb}$, on $12 \mathrm{pl}$, and contains $O L R 1$ as well as the gene encoding $\alpha 2$-macroglobulin $(A 2 M)$, while the more distal region near $50 \mathrm{Mb}$, on $12 \mathrm{ql}$, maps close to the genes encoding LRPI (low density lipoprotein-related protein 1) and TFCP2 (transcription factor CP2). All four of these genes have shown independent associations with AD risk, although the results for $A 2 M$ and LRPI have been controversial (for recent reviews see Bertram and Tanzi $^{3}$ and Saunders et $a l^{4}$ ).

For OLRl, Luedecking-Zimmer and colleagues first reported evidence of association with the 3' UTR SNP (rs1050283) in a white North American case-control sample of more than 1500 subjects. ${ }^{1}$ This association showed a strong interaction with $A P O E$ (apolipoprotein E) ع4-genotype, and led to opposite effects in subgroups stratified by $A P O E \varepsilon 4$. Specifically, APOE \&4-positive carriers of the TT-genotype had a 1.7-fold increased $\mathrm{AD}$ risk, whereas APOE \&4-negative carriers of the same genotype showed a significantly reduced $(\mathrm{OR}=0.7)$ risk for $\mathrm{AD}$. Performing electrophoretic mobility shift assays (EMSAs) indicated reduced binding of nuclear proteins related to the T-allele of this variant.

The more recently published results by Lambert et al were obtained on a large French case-control sample and a considerably smaller North American family-based sample, and also showed significant effects with this polymorphism. ${ }^{2}$ However, no APOE \&4-dependence was observed in either of the samples investigated in the latter study. Furthermore, these authors also saw a significant under-representation of the TT-genotype $v$ the CC/CT-genotypes, and this finding was interpreted as a risk effect in carriers of the C-allele $v$ noncarriers. Similar to the previous analyses, these genetic results were also supported by some functional data suggesting reduced binding of the T-allele to nuclear and cytoplasmic proteins as compared to the $\mathrm{C}$-allele as determined by EMSA. While no correlation between this polymorphism and cerebral $A \beta$ or tau load was observed in this study, there was evidence of a significantly reduced expression of $O L R I$ in carriers of the $\mathrm{C}$-allele carriers $v$ non-carriers. ${ }^{2}$

Our laboratory has previously tested several AD candidate genes on chromosome 12 for association with $\mathrm{AD}$ in one of the largest $\mathrm{AD}$ family samples collected to date, the NIMH Genetics Initiative Study sample. ${ }^{3-6}$ Of these, the strongest and most consistent signals observed were obtained with polymorphisms in $A 2 M$, which show a strong effect on $\mathrm{AD}$

\section{Key points}

- Chromosome 12 has been implicated to harbour one or more Alzheimer's disease (AD) loci by several laboratories using both linkage and association methods.

- Two studies recently observed genetic association with a 3' UTR single nucleotide polymorphism (SNP) in the gene encoding $O L R 1$, which is involved in the binding, internalisation, and degradation of oxidised lipoproteins.

- In a large sample of 437 multiplex AD families (1439 subjects) no evidence was observed favouring a genetic involvement of the same OLRI SNP in AD, nor was any linkage disequilibrium observed between this SNP and polymorphisms in the nearby $\mathrm{A} 2 \mathrm{M}$ gene, which show a strong disease association in the same sample.

- Our results suggest that the contribution of $O L R 1$ to $A D$ risk in the general population may be considerably smaller than previously suggested.

risk, predominantly in families with late-onset $\mathrm{AD}^{4}{ }^{5}$ Although $A 2 M$ maps about $1 \mathrm{Mb}$ pter of OLRl, it is conceivable that at least part of the independent association signals observed across our and the OLRI-positive studies is due to polymorphisms in linkage disequilibrium (LD) with genetic variants in these two genes. Thus, we have tested the OLRI SNP in the full NIMH sample, which is comprised of 1439 of individuals from 437 families in which all affected individuals had disease onset at $>50$ years. These include 994 affected individuals (mean age of onset $72.4 \pm 7.7$ years, range 50-97), 411 unaffecteds, and 34 with phenotype unknown. Pedigrees were classified as "late-onset" (320 pedigrees) when all sampled affecteds in each pedigree had onset ages of $>65$ years, and "early/mixed" otherwise. APOE genotyping was performed as described previously, ${ }^{7}$ and pedigrees were classified as "APOE- $44 / 4$ positive" when at least one affected individual per pedigree carried the $\varepsilon 4 / 4$ genotype (120 pedigrees), and as "APOE-\&4/4 negative" otherwise, and as "APOE- $\varepsilon 4$ positive" when at least one affected individual per pedigree carried the $\varepsilon 4$-allele (358

Abbreviations: $A 2 M, \alpha 2$-macroglobulin; $A D$, Alzheimer's disease; APOE, apolipoprotein $E ; C L R$, conditional logistic regression; EMSAs, electrophoretic mobility shift assays; LD, linkage disequilibrium; LRPI, low density lipoprotein-related protein 1; OLR I, oxidised LDL receptor 1 
pedigrees), and as "APOE-\&4 negative" otherwise (for more details on the NIMH sample see Blacker et $a l^{78}$ ).

Genotyping of rs1050283 was performed on all individuals using a high-efficiency fluorescence polarisation single-base extension (FP-SBE) detection assay, similar to that used for genotyping of most $A 2 M$ SNPs. ${ }^{4}$ Genotyping efficiency was at $96 \%$ and showed no discrepancies based on $\sim 10 \%$ internally duplicated samples. To test for association of this SNP with $\mathrm{AD}$, we used "FBAT" (v.1.4.2), a program for family-based association testing that allows for missing parental genotypes. ' Like the transmission-disequilibrium test (TDT) and other family-based association tests, this method is not susceptible to bias due to population admixture. For all analyses we used the empirical variance function (EV-FBAT) to account for the presence of linkage in the area, and an equal-weight offset correction to incorporate genotypes from both affected and unaffected individuals (see the FBAT website http://www.biostat.harvard.edu/ fbat/default.html for more details). The results of these analyses are shown in table 1 and did not reveal any evidence of association between this OLRI SNP and AD in the full NIMH sample, or in strata based on onset age or $A P O E$ genotype. In accordance with these findings, conditional logistic regression (CLR) analysis stratified on family showed no significant effects with either alleles or genotypes, nor did it detect any significant interactions of the OLRI SNP with age or APOE genotype (data not shown). Finally, none of four haplotype tagging variants in $A 2 M$ displayed any significant degree of pairwise LD with the OLRI SNPs (parameterised as $\mathrm{D}^{\prime}$, table 2), suggesting that LD is most likely not responsible for the previously observed independent $\mathrm{AD}$ associations reported for these genes. ${ }^{1245}$ This is in agreement with recent estimates indicating that detectable LD in outbred and heterogeneous populations such as these extends from one to several hundred $\mathrm{kb}$, but rarely beyond. ${ }^{10}$

Unfortunately, the reason for the conflicting findings regarding our negative and the previous positive results remains unclear. While our sample clearly has the power to detect effects of the magnitude discussed for OLRI, we were still unable to observe any evidence supporting a role of the analysed OLRI SNP (rs1050283) in our AD families. The same families, however, demonstrate strong and consistent association with several variants in the nearby $A 2 M$ gene. ${ }^{4}$ Finally, we did not detect evidence of LD between the OLRI SNP and four polymorphisms in $A 2 M$. There are at least three possible explanations for the discrepancies across studies. First, the previous positive associations between $O L R I$ and/or $A 2 M$ and $\mathrm{AD}$ are chance findings (type I errors). This seems unlikely because replications in multiple independent samples have been published for both genes. Second, both genes are in fact

Table 1 Association statistics for OLR1rs 1050283 and $A D$ in families of the NIMH sample

\begin{tabular}{|c|c|c|}
\hline & $\begin{array}{l}\text { Sample size, } \\
\boldsymbol{n}_{\text {families }}\left(\boldsymbol{n}_{\text {individuals }}\right)\end{array}$ & $\begin{array}{l}\text { Association (FBAT), } \\
\text { z score }(\mathbb{P})^{*}\end{array}$ \\
\hline Total & 437 (1439) & $0.4(0.655)$ \\
\hline$A P O E \varepsilon 4 / 4$-pos $\dagger$ & $120(453)$ & $0.6(0.568)$ \\
\hline APOE \&4-pos† & 358 (1213) & $1.0(0.313)$ \\
\hline APOE \&4/4-neg & $317(986)$ & $0.1(0.905)$ \\
\hline APOE \&4-neg†† & $79(226)$ & $-1.8(0.074)$ \\
\hline Late-onset & $120(996)$ & $0.2(0.856)$ \\
\hline Early/mixed-onset & 317 (443) & $0.5(0.615)$ \\
\hline
\end{tabular}

${ }^{*} z$ score for risk allele (C) of previous studies: frequency in total sample $=0.52$; tho significant interaction was observed between APOE$\varepsilon 4$ or $\varepsilon 4 / 4$ status and the OLRI SNP using CLR stratified on family (data not shown); the undertransmission of the C-allele in this stratum did not confer any significant decrease in risk for $A D$ as estimated by CLR (ORs

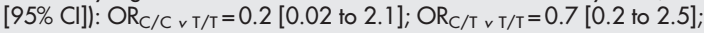
$O R_{\text {any }-C \vee T / T} 0.7$ [0.2 to 2.5$]$.
Table 2 Pairwise linkage disequilibrium of polymorphisms in $A 2 M$ and $O L R 1$

\begin{tabular}{lllllll}
\hline & A2M-UTR & \multicolumn{2}{l}{ A2M-12e } & A2M-15i & A2M-18i & OLR1 \\
\hline A2M-UTR & - & 0.72 & 1 & 0.94 & 0.02 \\
A2M-12e & $* * *$ & - & 0.86 & 0.78 & 0.11 \\
A2M-15i & $* * *$ & $* *$ & - & 1 & 0.05 \\
A2M-18i & $* * *$ & $* * *$ & $* * *$ & - & 0.06 \\
OLR1 & NS & NS & NS & NS & - \\
\hline
\end{tabular}

For a key to $A 2 M$ polymorphisms see Saunders et $a^{4}$. Cells above the diagonal represent $D^{\prime}$-values, and cells below the diagonal represent $P$ values (Fisher's exact test, two-sided: "NS" indicates $\mathrm{P}>0.05$; ${ }^{* *}$ indicates $\mathrm{P} \leqslant 0.01$; ${ }^{* * *}$ indicates $\mathrm{P} \leqslant 0.001$ ). LD statistics calculated with "2by2" (http://linkage.rockefeller.edu/ott/ linkutil.htm), based on haplotype frequencies in one affected individual per pedigree estimated by the FBAT program.

genuine AD susceptibility factors, but only exert small effects in the general population which are detectable in some study samples but not in others. ${ }^{124}$ This is supported by the fairly small effect sizes estimated for both genes (for example OLRI ORs about 2 for risk-allele carriers, ${ }^{12}$ and $A 2 M$ ORs between 1.8 and 3.5 in our sample $)^{4}$ as compared to the average ORs of about 3 for heterozygous and about 15 for homozygous carriers of the APOE 84 -allele. ${ }^{11}$ ) Third, as we do not find evidence of significant LD between the two genes, the independent findings with $O L R I$ in some $\mathrm{AD}$ populations, and $A 2 M$ in others could also reflect LD with variant(s) in a third gene located somewhere in the vicinity of these two candidates. In this context it is interesting to notice that several other members of the C-type lectin superfamily (to which OLRI belongs as well)-for example CLECI and 2 (C-type lectin-like receptors-1 and -2), CLL-1 (C-type lectin protein CLL-1), or LLT1 (lectin-like NK cell receptor-1)-map between OLRI and A2M (see: "UCSC Genome Browser" [July 2003 freeze]: http://genome.ucsc.edu/). More systematic LDbased mapping in additional $\mathrm{AD}$ samples is required to further elucidate the role of these and other proposed $\mathrm{AD}$ candidate genes on chromosome 12.

\section{ACKNOWLEDGEMENTS}

This work was sponsored by grants from the NIMH, NIA (ADRC) and the Alzheimer Association. LB was a fellow of the Deutsche Forschungsgemeinschaft (DFG) and now receives a translational fellowship of the Harvard Center for Neurodegeneration and Repair (HCNR), Core A

\section{Authors' affiliations}

L Bertram, M Parkinson, K Mullin, R Menon, R E Tanzi, Genetics and Aging Research Unit, MassGeneral Institute for Neurodegenerative Disease (MIND), Department of Neurology, Massachusetts General Hospital, Harvard Medical School, Charlestown, MA, USA

D Blacker, Gerontology Research Unit, Department of Psychiatry, Massachusetts General Hospital, Harvard Medical School, Charlestown, MA, USA

D Blacker, Department of Epidemiology, Harvard School of Public Health, Boston, MA, USA

Correspondence to: RE Tanzi, PhD, Genetics and Aging Research Unit, MGH-East (MIND), $11416^{\text {th }}$ St, Charlestown, MA 02129, USA;

tanzi@helix.mgh.harvard.edu

Received 28 November 2003

Accepted for publication 15 December 2003

\section{REFERENCES}

1 Luedecking-Zimmer E, DeKosky ST, Chen Q, Barmada MM, Kamboh MI. Investigation of oxidized LDL-receptor $1(\mathrm{OLR} 1)$ as the candidate gene for Alzheimer's disease on chromosome 12. Hum Genet 2002; $111(4-5): 443-51$.

2 Lambert JC, Luedecking-Zimmer E, Merrot S, Hayes A, Thaker U, Desai P Houzet A, Hermant X, Cottel D, Pritchard A, Iwatsubo T, Pasquier F, Frigard B, 
Conneally PM, Chartier-Harlin MC, DeKosky ST, Lendon C, Mann D, Kamboh MI, Amouyel P. Association of 3'-UTR polymorphisms of the oxidised LDL receptor 1 (OLR1) gene with Alzheimer's disease. J Med Genet 2003;40(6):424-30.

3 Bertram L, Tanzi RE. Dancing in the dark? The status of late-onset Alzheimer's disease genetics. J Mol Neurosci 2001;17(2):127-36.

4 Saunders AJ, Bertram L, Mullin K, Sampson AJ, Latifzai K, Basu S, Jones J, Kinney D, MacKenzie-Ingano L, Yu S, Albert MS, Moscarillo TJ, Go RC, Bassett SS, Daly MJ, Laird NM, Wang X, Velicelebi G, Wagner SL, Becker DK, Tanzi RE, Blacker D. Genetic association of Alzheimer's disease with multiple polymorphisms in alpha-2-macroglobulin. Hum Mol Genet 2003; 12(21):2765-76.

5 Blacker D, Wilcox MA, Laird NM, Rodes L, Horvath SM, Go RC, Perry R, Watson B, Bassett SS, Mclnnis MG, Albert MS, Hyman BT, Tanzi RE. Alpha-2 macroglobulin is genetically associated with Alzheimer disease. Nat Genet 1998; 19(4):357-60

6 Bertram L, Blacker D, Crystal A, Mullin K, Keeney D, Jones J, Basu S, Yhu S, Guenette S, Mclnnis M, Go R, Tanzi R. Candidate genes showing no evidence for association or linkage with Alzheimer's disease using family-based methodologies. Exp Gerontol 2000;35(9-10):1353-1361.
7 Blacker D, Haines JL, Rodes L, Terwedow H, Go RC, Harrell LE, Perry RT, Bassett SS, Chase G, Meyers D, Albert MS, Tanzi R. ApoE-4 and age at onset of Alzheimer's disease: the NIMH genetics initiative. Neurology 1997:48(1): 139-47.

8 Blacker D, Bertram L, Saunders AJ, Moscarillo TJ, Albert MS, Wiener $\mathrm{H}$ Perry RT, Collins JS, Harrell LE, Go RC, Mahoney A, Beaty T, Fallin MD, Avramopoulos D, Chase GA, Folstein MF, Mclnnis MG, Bassett SS, Doheny KJ, Pugh EW, Tanzi RE. Results of a high-resolution genome screen of 437 Alzheimer's disease families. Hum Mol Genet 2003; 12(1):23-32

9 Rabinowitz D, Laird N. A unified approach to adjusting association tests for population admixture with arbitrary pedigree structure and arbitrary missing marker information. Hum Hered 2000;50(4):211-23.

10 Wall JD, Pritchard JK. Haplotype blocks and linkage disequilibrium in the human genome. Nat Rev Genet 2003;4(8):587-97.

11 Farrer LA, Cupples LA, Haines JL, Hyman B, Kukull WA, Mayeux R, Myers RH, Pericak-Vance MA, Risch N, van Duijn CM. Effects of age, sex, and ethnicity on the association between apolipoprotein $\mathrm{E}$ genotype and Alzheimer disease. A meta-analysis. APOE and Alzheimer Disease Meta Analysis Consortium. JAMA 1997;278(16):1349-56.

\section{$\mathrm{ECHO}$}

\section{Modifier genes and familial adenomatous polyposis coli}

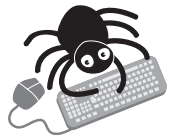

Please visit the Journal of

Medical

Genetics

website [www. jmedgenet. com] for a link to the full text of this article.
$T$ he number of adenomatous polyps in somebody with familial adenomatous polyposis (FAP) is influenced by the position of the germline mutation in the APC gene. This, however, does not explain the variability within families which is consistent with the effect of modifier genes. Such genes are known to affect disease severity in a mouse model. Several common polymorphisms may be associated with increased risk of colorectal cancer and could act as APC modifier genes. Researchers in London, UK have studied polymorphisms in eight genes (MTHFR, NAT1, NAT2, GSTM, GSTT, cyclin D1, E-cadherin, and $A P C$ ) in relation to severity of FAP (number of polyps per colectomy specimen standardised for colon size).

DNA samples (from blood, established cell line, or fixed normal tissue) were obtained from 151 patients from 51 families with established classical FAP. The relationship between polyp count and genotype at each polymorphic site was assessed, making allowance for the position of the germline APC mutation. Among all 151 patients severe disease (high polyp count) was associated with variations in the $\mathrm{N}$-acetyltransferase genes; more specifically with the absence of the $N A T 1{ }^{*} 10$ genotype and the presence of both $N A T I *$ non- 10 and NAT2*fast genotypes. There was a weak association between severe disease and the APCT1493C allele. None of the other polymorphisms analysed was associated with disease severity. Among patients with germline mutations in the "mutation cluster region" (MCR) severe disease was associated with the presence of $N A T 2 *$ fast alleles.

Alleles at NAT1 and/or NAT2 loci on chromosome 8p22 may act as disease severity modifiers in patients with FAP and may explain a twofold variation in polyp number.

\ Gut 2004;53:271-276. 